\title{
Modeling of Constructions' Structurally Nonlinear Oscillations Using Chebyshev's Polynomials
}

\author{
Sergei Yu. GRIDNEV*, Yuri I. SKALKO**, Alexandr O. SHIMANOVSKY*** \\ *Voronezh State Technical University, Moskovsky av., 14, Voronezh, 394026, Russia; Moscow Institute of Physics and \\ Technology (State University), Institutskiy per., 9, Dolgoprudny, Moscow Region, 141701, Russia, \\ E-mail: gridnev_s_y@rambler.ru \\ **Institute of Physics and Technology (State University), Institutskiy per. 9, Dolgoprudny, Moscow Region, 141701, \\ Russia,E-mail: skalko@mail.mipt.ru \\ ***Belarusian State University of Transport, Kirova, 34, Gomel, 246653, Belarus, E-mail: tm.belsut@ gmail.com \\ cross $^{\text {ref }}$ http://dx.doi.org/10.5755/j01.mech.26.4.24214
}

\section{Introduction}

Modeling of oscillations of various dynamic systems is a constructive-nonlinear problem in some cases. They are the systems with one-way connections, various designs with enabling and disabling connections, constructions after destruction of one or several elements with the calculation scheme change, mechanisms with gaps and backlashes, constructions with elastic connections and preliminary compression by a construction body own weight or motion limiters, etc. The differential equations in partial derivatives with discontinuous boundary conditions are used in mathematical model for the description of behavior of such systems. Such constructions are floating bridges of continuous system with additional limiting rigid supports. On the ends of these supports river parts of the bridge rely only in the case of passing mobile loading. Generally, in the case of mobile loading motion along the floating bridges of not continuous system the greatest bending moments and lowering arise at their ends. It is impractical to increase buoyancy of all bridge to get the admissible free end lowering because the middle part of the bridge will have excess margins of buoyancy and durability. Therefore, in order to avoid the floating bridge end lowering there are used the limiting rigid supports under the beginning and the end of river-transitional parts connection in modern floating bridges.

In the absence of mobile loading on the floating bridge there are available vertical gaps between it's span and rigid supports. During the movement of the loading along the river or transitional parts of the span, vertical gaps at one or both sides change and, generally, the ends of the bridge river part can alternately or simultaneously lean on rigid support (Fig. 1).

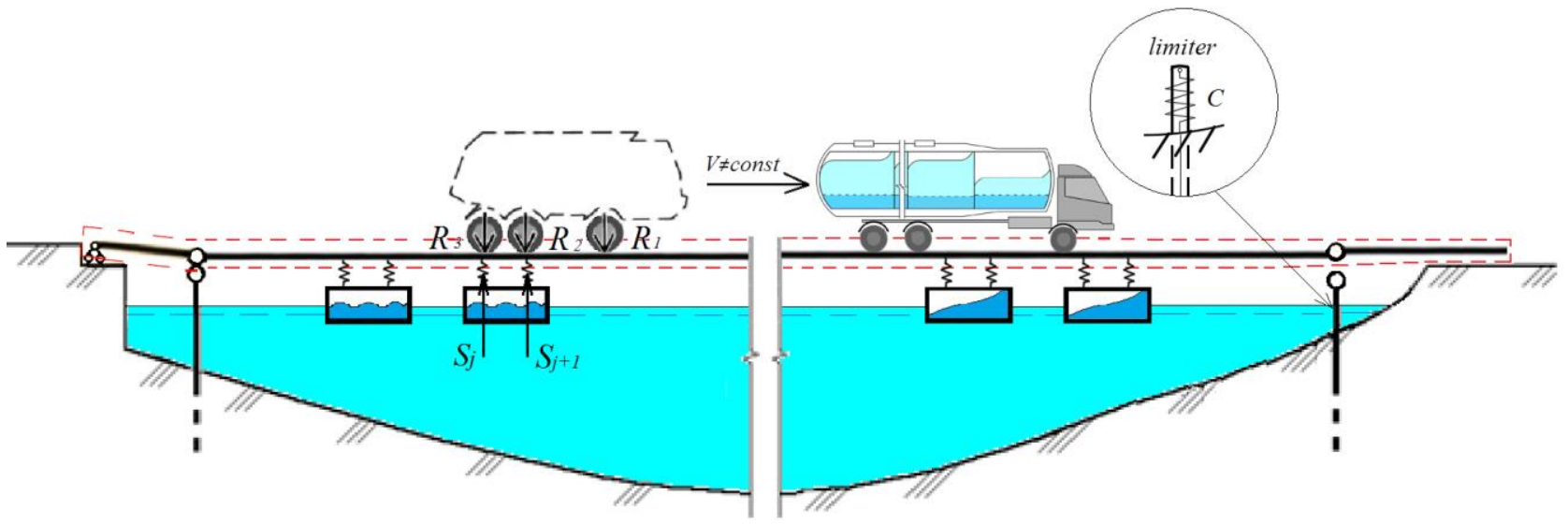

Fig. 1 Span structure (marked by dots) in the general oscillatory system "Car + floating bridge of continuous system with additional limiting rigid supports"

Practically a vertical gap between the bridge span and rigid support is appointed by not less than a half of draft of the bridge middle under the action of the estimated passing loading weight [1]. The dynamic influence of mobile loading on the span middle draft at the modeling of the floating bridge oscillations as not continuous system with additional limiting rigid support has an important practical value. The influence of floating bridge limiting supports on stress strain behavior was earlier estimated only at static loading [1]. Inertia forces of the mobile loading and the bridge, and also the influence of back link weren't considered. The analysis of features of floating bridge oscillations in case of mobile loading considering the nonlinear effects of end supports operation is urgent and important at the mobile loading weight increase. There are not yet invented the strict algorithms for dynamic calculations of bridge bearing structures oscillations together with the vehicle moving on it, taking into account change of the calculation scheme depending on a mobile loading position.

Despite of a large number of publications devoted to problems of one-way connections it is necessary to develop simple and effective method and calculation algorithm for the solution of the considered task. Complexity of its implementation consists in the solution of differential 
equations with discontinuous boundary conditions due to the unknown time of boundary conditions change.

Statement of a problem of constructive nonlinearity accounting at floating bridges oscillations considering the additional limiting rigid supports is described in [2] and it is solved by the one-dimensional piecewise linear models with concentrated parameters. The results are extended to a bridge span which is a distributed system.

In recent years there was published a number of works devoted to investigation of the floating bridges behavior at static and dynamic loadings. It should be noted the active research work in this field, made by Fu S., Cui W., Chen X., Wang C., Lin Z.-M. and others. Papers [3-6] describe the new dynamic calculation models used for the analysis of oscillations of floating and continuous bridges systems in a deterministic setting, depending on the moving load size and velocity. Work [7] studies the effect of different water depths on the oscillations features. Paper [8] determines frequencies and forms of free oscillations of bridges, considering the impact of bridge horizontal cables fixing and torsion oscillations. In paper [9] floating bridge oscillations are investigated, taking into account the partial filling of separate supports with water. The problem is solved by the stochastic finite element method. The works $[10,11]$ consider the characteristics of the wave loading, magnitudes of the hydrodynamic coefficients and seismic loading. To perform the research there are used the finite element method and a number of numerical methods $[12,13]$. Such an interest shows their wide use in the practice of transportation construction. There are not found the publications on the calculation of floating bridges of continuous system with limiting rigid supports under the action of the moving loading, which are the structural and non-linear systems. Papers [14-16] are devoted to the study of structural and nonlinear oscillations of constructions. The solution of some problems of building structures static calculation can be obtained in analytic form that allows to study the impact of various construction parameters in an explicit form [17]. This work is performed on the basis of the results obtained in $[2,18]$ and it is their continuation and development.

The purpose of this work is to develop a technique for modeling structural and nonlinear oscillations of building structures on the example of a span of a floating bridge of continuous system with limiting rigid supports under the moving loading action. To achieve this, it is necessary to develop a computational algorithm for solving partial differential equations with discontinuous boundary conditions.

\section{Calculation scheme and mathematical model}

The structural-nonlinear oscillations of the floating bridge span are considered. The problem is solved in a flat substitution, that corresponds to the case of a car moving along the axis of a bridge or roadway with only one traffic lane.

A model of an elastic rod of length $l$ with free ends previously considered in $[2,18]$ was taken as a calculation scheme. In this model the rod is under the action of a combination of concentrated forces from the dynamic tire pressures $R_{k}(t)$ of a three-axle vehicle, forces in the linings under the floating supports $S_{j}(t)$ and forces $P_{l}(t)$ and $P_{r}(t)$ acting the rod from the left and right side of the transition parts, respectively (Fig. 2).

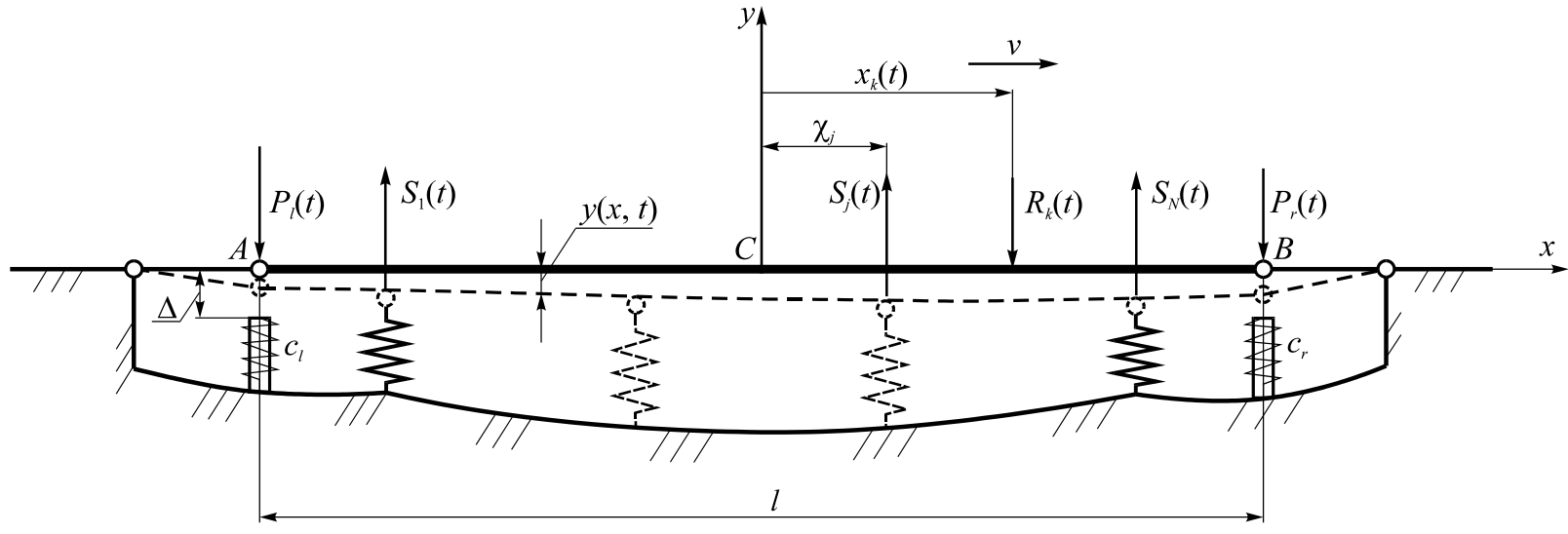

Fig. 2 Scheme of the floating bridge

Bending oscillations of an elastic rod (bridge span model) in the vertical plane are described by a partial differential equation of the fifth (or fourth if the internal friction is neglected) order relative to the coordinate $y(x, t)$, measured from the rod position in equilibrium. If the origin coincides with the mass center position $C$ of the rod, there are no forces $R_{k}(t)$ than the equation is:

$$
\mu \frac{\partial^{2} y(x, t)}{\partial t^{2}}+\left(1+\aleph \frac{\partial}{\partial t}\right) E J \frac{\partial^{4} y(x, t)}{\partial x^{4}}-\sum_{j=1}^{N} S_{j}(t) \delta\left(\chi_{j}\right)+\sum_{k=1}^{3} R_{k}(t) \delta\left(x_{k}\right)+P_{l}(t) \delta\left(-\frac{l}{2}\right)+P_{r}(t) \delta\left(\frac{l}{2}\right)=-\mu g
$$

where: $\mu$ is linear rod mass; $\aleph$ is attenuation coefficient of bending strain due to internal friction; $E$ is material elasticity modulus; $J$ is moment of inertia the rod's cross section; $N$ is number of floating supports; $\delta(x)$ is Dirac delta function, allowing to take into account the concentrated forces action in the equation with distributed parameters; $\chi_{j}$ and $x_{k}$ are coordinates of forces $S_{j}(t)$ and $R_{k}(t)$ application points correspondingly.

It should be noted that $S_{j}=\frac{M g}{N}+S_{j}^{*}$, where $M$ is 
bridge span mass. The first term describes the loading of the intermediate supports from the span sole weight in equilibrium. The total force from all the intermediate supports is equal to the total weight of the bridge. The second term reflects the additional force from the intermediate support when the bridge leaves the equilibrium position.

Under the action of forces $R_{k}(t)$ it should be taken into account the possibility of gaps closing in the case of span extreme point displacement exceeding $\Delta$ (Fig. 2). In this case, additionally the forces $F_{l}(t)$ and $F_{r}(t)$ start to act the elastic rod from the limiting supports. The position when the span is in contact with the limiting supports depends not only on the bending deformations $y(x, t)$, but also on the displacement of the bridge as an absolutely rigid body, which is determined by the coordinate of the mass center $z(t)$ and the rotation angle $\varphi(t)$ (Fig. 3).

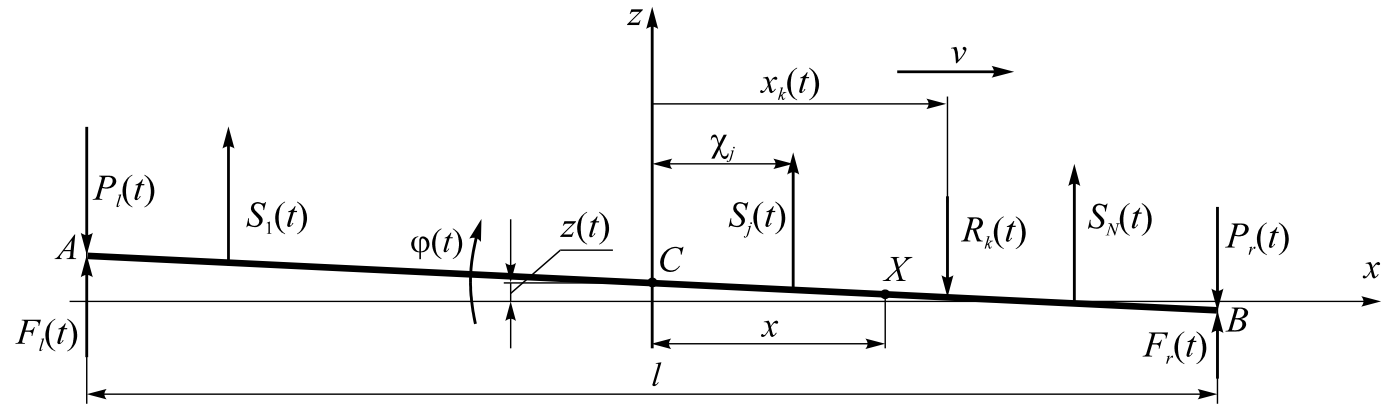

Fig. 3 The span displacement scheme for the floating bridge modeled as the rigid body

In the case of small displacements at $\tan \varphi=\varphi$, the movement of the span as a solid body is described by

the equations:

$$
\left\{\begin{array}{l}
M \frac{d^{2} z}{d t^{2}}+\sum_{i=1}^{3} R_{k}(t)-\sum_{j=1}^{N} S_{j}(t)+P_{l}(t)-F_{l}(t)+P_{r}(t)-F_{r}(t)+m g=0 \\
J_{b} \frac{d^{2} \varphi}{d t^{2}}-\sum_{i=1}^{3} R_{k}(t) x_{k}(t)+\sum_{j=1}^{N} S_{j}(t) \chi_{j}+\left(P_{r}(t)-F_{r}(t)-P_{l}(t)+F_{l}(t)\right) \frac{l}{2}=0
\end{array} .\right.
$$

where: $J_{b}$ is central moment of inertia of the rod as a solid.

The initial conditions for the differential equations system (2) are:

$$
z(0)=0 ; \frac{d z}{d t}(0)=0 ; \varphi(0)=0 ; \frac{d \varphi}{d t}(0)=0 .
$$

Thus, the full vector of generalized coordinates determining the position of a random span point has the form $\boldsymbol{Z}=[y(x, t), z(t), \phi(t)]^{T}$.

Then there was created a mathematical model of an elastic thin rod bending movements in the coordinate system associated with this rod, as with a rigid body.

This coordinate system is non-inertial, therefore, in the motion Eq. (1) it is necessary to take into account the distributed inertia forces acting at a point with a coordinate $x$ additionally. As the vertical coordinate of point $X$ is connected with the generalized coordinates $z$ and $\varphi$ by the relation $z_{X}=z-x \varphi$ (Fig. 3), then the corresponding inertia force $\Phi=-\mu \frac{\partial^{2} z_{X}}{\partial t^{2}}=-\mu\left(\frac{\partial^{2} z}{\partial t^{2}}-x \frac{\partial^{2} \varphi}{\partial t^{2}}\right)$.

Therefore, the Eq. (1) takes the form:

$$
\mu \frac{\partial^{2} y}{\partial t^{2}}+\left(1+\aleph \frac{\partial}{\partial t}\right) E J \frac{\partial^{4} y}{\partial x^{4}}+\mu \frac{\partial^{2} z}{\partial t^{2}}-\mu x \frac{\partial^{2} \varphi}{\partial t^{2}}=-\mu g+\sum_{j=1}^{N} S_{j} \delta\left(\chi_{j}\right)-\sum_{k=1}^{3} R_{k} \delta\left(x_{k}\right)+\left(F_{l}-P_{l}\right) \delta\left(-\frac{l}{2}\right)+\left(F_{r}-P_{r}\right) \delta\left(\frac{l}{2}\right)
$$

The new variable $u(x, t)=y(x, t)+z(t)-x \varphi(t)$ describes the displacements of the rod points at a time $t$ in a fixed coordinate system in the case of rod small angular deviations from the equilibrium position.

To determine the values of forces $F_{l}(t)$ and $F_{r}(t)$ from the limiting supports the Heaviside function $\theta(-\Delta-u)$ was applied. This function is equal to 0 if its argument is negative, and 1 if it is positive. Using the Heaviside function, there could be uniformly written down the boundary conditions both for the case of opened and closed gaps. The changing of boundary conditions takes place when the function argument changes its sign, i.e., when $u=-\Delta$. It is considered that the uttermost limiting supports are not absolutely rigid, and their action can be modeled using elastic springs with large stiffness factors $c_{l}$ and $c_{r}$. In this case: $F_{l}=\left.\theta(-\Delta-u) c_{l}(-\Delta-u)\right|_{-\frac{l}{2}}$;

$$
F_{r}=\left.\theta(-\Delta-u) c_{r}(-\Delta-u)\right|_{\frac{l}{2}} .
$$

The considered approach allows to consider the floating bridge span as a rod with free ends even at its contact with the limiting supports. In this case, the internal transverse forces and bending torques at the rod ends are equal to zero. Consequently, the elastic thin rod motion can be written in the form of an initial-boundary value problem for a partial differential equation, taking into account expressions (3): 


$$
\left\{\begin{array}{l}
\mu \frac{\partial^{2} u}{\partial t^{2}}+\left(1+\aleph \frac{\partial}{\partial t}\right) E J \frac{\partial^{4} u}{\partial x^{4}}=-\mu g+\sum_{j=1}^{N} S_{j} \delta\left(\chi_{j}\right)-\sum_{k=1}^{3} R_{k} \delta\left(x_{k}\right)+\left(F_{l}-P_{l}\right) \delta\left(-\frac{l}{2}\right)+\left(F_{r}-P_{r}\right) \delta\left(\frac{l}{2}\right), \\
u(x, 0)=0 ; \quad \frac{\partial u}{\partial t}(x, 0)=0 ; \quad \frac{\partial^{2} u}{\partial x^{2}}\left(-\frac{l}{2}, t\right)=\frac{\partial^{2} y}{\partial x^{2}}\left(-\frac{l}{2}, t\right)=0 ; \quad \frac{\partial^{3} u}{\partial x^{3}}\left(-\frac{l}{2}, t\right)=\frac{\partial^{3} y}{\partial x^{3}}\left(-\frac{l}{2}, t\right)=0 \\
\frac{\partial^{2} u}{\partial x^{2}}\left(\frac{l}{2}, t\right)=\frac{\partial^{2} y}{\partial x^{2}}\left(\frac{l}{2}, t\right)=0 ; \quad \frac{\partial^{3} u}{\partial x^{3}}\left(\frac{l}{2}, t\right)=\frac{\partial^{3} y}{\partial x^{3}}\left(\frac{l}{2}, t\right)=0 .
\end{array}\right.
$$

The application of the Galerkin method and finite differences for the Eq. (1) made it possible to obtain stable solutions only for a certain range of stiffness coefficients of limiting supports. Their increase in order to obtain more accurate results led to the algorithm stability loss. Therefore, in this work, there was applied the apparatus of generalized functions, which are continuous linear functionals on the space of basic functions $D$, consisting of infinitely differentiable smooth compactly supported functions [19]. A regular generalized function can be associated with each integrated ordinary function $f(x)$ and this function acts a arbitrary test function $\psi(x) \in D$ according to the rule:

$$
(f, \psi)=\int f \psi d x=I .
$$

The solution will be searched in the class of four times continuous differentiable generalized functions $u(t, x) \in C^{4}\left(-\frac{l}{2}, \frac{l}{2}\right)$.

For the convenience of further description:

$$
q=\sum_{j=1}^{N} S_{j} \delta\left(\chi_{j}\right)-\sum_{k=1}^{3} R_{k} \delta\left(x_{k}\right)+\left(F_{l}-P_{l}\right) \delta\left(-\frac{l}{2}\right)+\left(F_{r}-P_{r}\right) \delta\left(\frac{l}{2}\right)=\sum_{s=1}^{N+5} Q_{s} \delta\left(x_{s}\right)
$$

Here: $q(x)$ can be considered as a variation law of the external load distributed along the rod length, corresponding to the action of concentrated forces.
Then the Eq. (4) will be rewritten as:

$$
\mu \frac{\partial^{2} u}{\partial t^{2}}+\left(1+\aleph \frac{\partial}{\partial t}\right) E J \frac{\partial^{4} u}{\partial x^{4}}=-\mu g+q .
$$

According to the definition (5) there follows that:

$$
I=\left(\mu \frac{\partial^{2} u}{\partial t^{2}}+\left(1+\aleph \frac{\partial}{\partial t}\right) E J \frac{\partial^{4} u}{\partial x^{4}}, \psi(x)\right)=\int_{-l / 2}^{l / 2} \mu \frac{\partial^{2} u}{\partial t^{2}} \psi d x+\left(1+\aleph \frac{\partial}{\partial t}\right) E J \int_{-l / 2}^{l / 2} u \frac{\partial^{4} \psi}{\partial x^{4}} d x .
$$

After double integration in parts:

$$
I=\int_{-l / 2}^{l / 2} \mu \frac{\partial^{2} u}{\partial t^{2}} \psi d x+\left(1+\aleph \frac{\partial}{\partial t}\right) E J \int_{-l / 2}^{l / 2} \frac{\partial^{2} u}{\partial x^{2}} \frac{\partial^{2} \psi}{\partial x^{2}} d x+\left.\left(1+\aleph \frac{\partial}{\partial t}\right) E J\left(\frac{\partial^{3} \psi}{\partial x^{3}} u-\frac{\partial^{2} \psi}{\partial x^{2}} \frac{\partial u}{\partial x}\right)\right|_{-l / 2} ^{l / 2}
$$

And one more double integration in parts:

$$
\begin{aligned}
& I=\int_{-l / 2}^{l / 2} \mu \frac{\partial^{2} u}{\partial t^{2}} \psi d x+\left(1+\aleph \frac{\partial}{\partial t}\right) E J \int_{-l / 2}^{l / 2} \frac{\partial^{4} u}{\partial x^{4}} \psi d x+\left.\left(1+\aleph \frac{\partial}{\partial t}\right) E J\left(\frac{\partial^{3} \psi}{\partial x^{3}} u-\frac{\partial^{2} \psi}{\partial x^{2}} \frac{\partial u}{\partial x}+\frac{\partial \psi}{\partial x} \frac{\partial^{2} u}{\partial x^{2}}-\psi \frac{\partial^{3} u}{\partial x^{3}}\right)\right|_{-l / 2} ^{l / 2}= \\
& =\int_{-l / 2}^{l / 2}\left\{\mu \frac{\partial^{2} u}{\partial t^{2}}+\left(1+\aleph \frac{\partial}{\partial t}\right) E J \frac{\partial^{4} u}{\partial x^{4}}\right\} \psi d x+\left.\left(1+\aleph \frac{\partial}{\partial t}\right) E J\left(\frac{\partial^{3} \psi}{\partial x^{3}} u-\frac{\partial^{2} \psi}{\partial x^{2}} \frac{\partial u}{\partial x}+\frac{\partial \psi}{\partial x} \frac{\partial^{2} u}{\partial x^{2}}-\psi \frac{\partial^{3} u}{\partial x^{3}}\right)\right|_{-l / 2} ^{l / 2} .
\end{aligned}
$$

Taking into account the Eq. (7) and boundary conditions (4), it turns out:

$$
\begin{aligned}
& I=\int_{-l / 2}^{l / 2}(-\mu g+q) \psi d x+\left.\left(1+\aleph \frac{\partial}{\partial t}\right) E J\left(\frac{\partial^{3} \psi}{\partial x^{3}} u-\frac{\partial^{2} \psi}{\partial x^{2}} \frac{\partial u}{\partial x}\right)\right|_{-l / 2} ^{l / 2}= \\
& =-\int_{-l / 2}^{l / 2} \mu g \psi d x+\sum_{s=1}^{N+5} \int_{-l / 2}^{l / 2} Q_{s} \delta\left(x_{s}\right) \psi d x+\left.\left(1+\aleph \frac{\partial}{\partial t}\right) E J\left(\frac{\partial^{3} \psi}{\partial x^{3}} u-\frac{\partial^{2} \psi}{\partial x^{2}} \frac{\partial u}{\partial x}\right)\right|_{-l / 2} ^{l / 2}
\end{aligned}
$$

As $\int_{-l / 2}^{l / 2} Q_{s} \delta\left(x_{s}\right) \psi d x=Q_{s} \psi\left(x_{s}\right)$, there follows that:

$$
I=-\int_{-l / 2}^{l / 2} \mu g \psi d x+\sum_{s=1}^{N+5} Q_{s} \psi\left(x_{s}\right)+\left.\left(1+\aleph \frac{\partial}{\partial t}\right) E J\left(\frac{\partial^{3} \psi}{\partial x^{3}} u-\frac{\partial^{2} \psi}{\partial x^{2}} \frac{\partial u}{\partial x}\right)\right|_{-l / 2} ^{l / 2}
$$


After equating (8) and (9) it follows that:

$$
\int_{-l / 2}^{l / 2} \mu \frac{\partial^{2} u}{\partial t^{2}} \psi d x+\left(1+\aleph \frac{\partial}{\partial t}\right) E J \int_{-l / 2}^{l / 2} \frac{\partial^{2} u}{\partial x^{2}} \frac{\partial^{2} \psi}{\partial x^{2}} d x=-\int_{-l / 2}^{l / 2} \mu g \psi d x+\sum_{s=1}^{N+5} Q_{s} \psi\left(x_{s}\right) .
$$

Taking into consideration (6), there is finally obtained the equation to determine the function $u(x, t)$ using a

random infinitely-differentiable finite basic function $\psi(x)$.

$$
\begin{aligned}
& \mu \int_{-l / 2}^{l / 2} \frac{\partial^{2} u}{\partial t^{2}} \psi d x=-\left(1+\aleph \frac{\partial}{\partial t}\right) E J \int_{-l / 2}^{l / 2} \frac{\partial^{2} u}{\partial x^{2}} \frac{\partial^{2} \psi}{\partial x^{2}} d x-\mu g \int_{-l / 2}^{l / 2} \psi d x+ \\
& +\sum_{j=1}^{N} S_{j} \psi\left(\chi_{j}\right)-\sum_{k=1}^{3} R_{k} \psi\left(x_{k}\right)+\left(F_{l}-P_{l}\right) \psi\left(-\frac{l}{2}\right)+\left(F_{r}-P_{r}\right) \psi\left(\frac{l}{2}\right) .
\end{aligned}
$$

Compared to Eq. (4), the obtained form of equation no longer contains the Dirac $\delta$-function and allows to operate with finite values of quantities. The derived equation is taken as the basis for constructing the computational algorithm.

\section{Creation of computational algorithm}

The solution will be searched in the form $u(x, t)=\sum_{n=1}^{K} H_{n}(x) T_{n}(t)$, where the Chebyshev polynomials of the first kind, order $n$ from 1 to $K$ are the basis function $H_{n}(x)$. As the test functions there are set the polynomials $\psi^{m}(x)=H^{m}(x)$, conjugated to $H_{n}(x)$, i.e. defined by the condition $\int_{-l / 2}^{l / 2} H^{m} H_{n} d x=\left\{\begin{array}{l}1, \text { if } m=n \\ 0, \text { if } m \neq n\end{array}\right.$.

Hereinafter, the indices related to the basis function are indicated below, and to the main one - above.

Then the substitution in (10) gives:

$$
\begin{aligned}
& \mu \sum_{n=1}^{K}\left(\frac{\partial^{2} T_{n}}{\partial t^{2}} \int_{-l / 2}^{l / 2} H^{m} H_{n} d x\right)=-\left(1+\aleph \frac{\partial}{\partial t}\right) E J \sum_{n=1}^{K}\left(T_{n} \int_{-l / 2}^{l / 2} \frac{\partial^{2} H^{m}}{\partial x^{2}} \frac{\partial^{2} H_{n}}{\partial x^{2}} d x\right)-\mu g \int_{-l / 2}^{l / 2} H^{m} d x+ \\
& +\frac{M g}{N} \sum_{j=1}^{N} H^{m} d x+\sum_{j=1}^{N}\left(\left.S_{j}^{*}\left(\sum_{n=1}^{K} T_{n} H_{n}\right) H^{m}\right|_{x=\chi_{j}}\right)-\sum_{k=1}^{3} R_{k} H^{m}\left(x_{k}\right)-P_{l} H^{m}\left(-\frac{l}{2}\right)-P_{r} H^{m}\left(\frac{l}{2}\right)+ \\
& +\left.\theta\left(-\Delta-T_{n} H_{n}\right) c_{l}\left(-\Delta-T_{n} H_{n}\right) H^{m}\right|_{x=-l / 2}+\left.\theta\left(-\Delta-T_{n} H_{n}\right) c_{r}\left(-\Delta-T_{n} H_{n}\right) H^{m}\right|_{x=l / 2} .
\end{aligned}
$$

To make the equation more compact we will use:

$$
\begin{aligned}
& \boldsymbol{T}(t)=[T]_{n}(t)=T_{n}(t) ;[\boldsymbol{H H}]=[H H]_{n}^{m}=\int_{-l / 2}^{l / 2} \mu H^{m} \\
& {\left[\mathbf{H}_{d} \mathbf{H}_{d}\right]=\left[H_{d} H_{d}\right]_{n}^{m}=} \\
& =\left(1+\aleph \frac{\partial}{\partial t}\right) E J \int_{-l / 2}^{l / 2} \frac{\partial^{2} H^{m}}{\partial x^{2}} \frac{\partial^{2} H_{n}}{\partial x^{2}} d x ; \\
& \mathbf{W}(\mathbf{T})=W^{m}\left(T_{n}\right)=\sum_{j=1}^{N}\left(\left.S_{j}^{*}\left(\sum_{n=1}^{K} T_{n} H_{n}\right) H^{m}\right|_{x=\chi_{j}}\right)+ \\
& +\left.\theta\left(-\Delta-T_{n} H_{n}\right) c_{l}\left(-\Delta-T_{n} H_{n}\right) H^{m}\right|_{x=-l / 2}+ \\
& +\left.\theta\left(-\Delta-T_{n} H_{n}\right) c_{r}\left(-\Delta-T_{n} H_{n}\right) H^{m}\right|_{x=l / 2} ; \\
& \mathbf{f}=f^{m}=-\int_{-l / 2}^{l / 2} \mu g H^{m} d x+\frac{M g}{N} \sum_{j=1}^{N} H^{m} d x- \\
& -\sum_{k=1}^{3} R_{k} H^{m}\left(x_{k}\right)-P_{l} H^{m}\left(-\frac{l}{2}\right)-P_{r} H^{m}\left(\frac{l}{2}\right) .
\end{aligned}
$$

Then the system of equations can be rewriting as: $[\mathbf{H H}] \frac{d^{2} \mathbf{T}}{d t^{2}}=-\left[\mathbf{H}_{d} \mathbf{H}_{d}\right] \mathbf{T}+\mathbf{F}(\mathbf{T})+\mathbf{f}$.

Multiplying by the inverse matrix $[\mathbf{H H}]$, it is obtained $\frac{d^{2} T_{n}}{d t^{2}}=-\sum_{n=1}^{K} A_{n}^{m} T_{n}+B^{m}\left(T_{n}\right)+P^{m}(t)$, where:

$$
A_{n}^{m}=[\mathbf{H H}]^{-1}\left[\mathbf{H}_{d} \mathbf{H}_{d}\right] ; B^{m}\left(T_{n}\right)=[\mathbf{H H}]^{-1} \mathbf{W}(\mathbf{T}) ;
$$

$$
P^{m}(t)=[\boldsymbol{H} \boldsymbol{H}]^{-1} \boldsymbol{f} .
$$

Taking into account the initial conditions: $T_{n}(t=0)=0 ; \frac{d T^{n}}{d t}(t=0)=0$, we obtain the Cauchy problem with respect to unknown functions $T_{n}$. Substitution of the ordinary differential equations resulting system solution in the expression $u(x, t)=\sum_{n=1}^{K} H_{n}(x) T_{n}(t)$ gives the form of the floating bridge span under the action of dynamic loads.

If necessary, the found function can be substituted into the Eq. (2), this will allow to find the laws of the $z$ and $\varphi$ generalized coordinates changing and the beam elastic axis deformations $y(x)$.

\section{Computational results}

Based on the developed algorithm, a computer program was created. The solution to the Cauchy problem was implemented in the Matlab environment using the built-in module for solving systems of ordinary differential equations, based on the one-step explicit Runge-Kutta method of the $2^{\text {nd }}$ and $4^{\text {th }}$ order with automatic step selection. A wide range of numerical investigations was performed to analyze the effect of bending stiffness, the size of the gap between the limiting supports and the ends of the rod, the loading velocity and its value on the ampli- 
tudes and features of the vibrations.

For example, there are presented some results of numerical calculations for a rod of length $l=42.5 \mathrm{~m}$, linear mass $\mu=888 \mathrm{~kg} / \mathrm{m}$, the cross section axial moment of inertia $J=0.0050936 \mathrm{~m}^{4}$. The rod relied on five elastic bonds with a step of $9.8 \mathrm{~m}$. It was considered that a force moves along the beam at a constant velocity. The force varied by the harmonic law with respect to the static value $P_{0}=2 \mathrm{kN}$ with a frequency $\omega=4 \pi \mathrm{c}^{-1}$ and amplitude $A=0.3 \mathrm{kN}$, which simulates the effects of the car wheels.

To illustrate the numerical results, the Fig. 4 shows the graphs of the beam leftmost point vertical displacements for various stiffness coefficients of the limiting supports when the mobile loading moves at a $15 \mathrm{~km} / \mathrm{h}$ velocity and the gaps value $\Delta=0.15 \mathrm{~m}$.

The seventh-order polynomials were used for the calculations. At high rigidity of the supports, the discontinuities of displacements are not observed, this confirms the efficiency of the algorithm. The computational results were very close to the observed in practice ones. It should be noted that the duration of the floating bridge contact with the limiting support is practically independent of its stiffness coefficient.

Fig. 5 shows a deformed view of the rod at the moment of rod relying on the left limiting support at different stiffness coefficients of these supports. A certain excess of the gap $\Delta$ is caused by the deformation of the limiting supports.

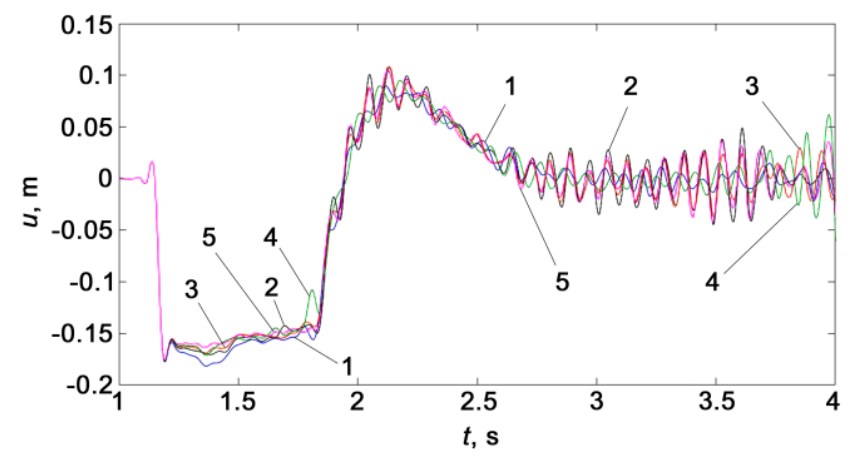

Fig. 4 Dependence of the beam leftmost point vertical displacements for the different stiffness coefficients of the limiting supports on time: $1-c_{l}=c_{r}==12$ $\mathrm{MN} / \mathrm{m} ; 2-c_{l}=c_{r}=22 \mathrm{MN} / \mathrm{m} ; 3-c_{l}=c_{r}==32 \mathrm{MN} / \mathrm{m}$; $4-c_{l}=c_{r}=42 \mathrm{MN} / \mathrm{m} ; 5-c_{l}=c_{r}=120 \mathrm{MN} / \mathrm{m}$

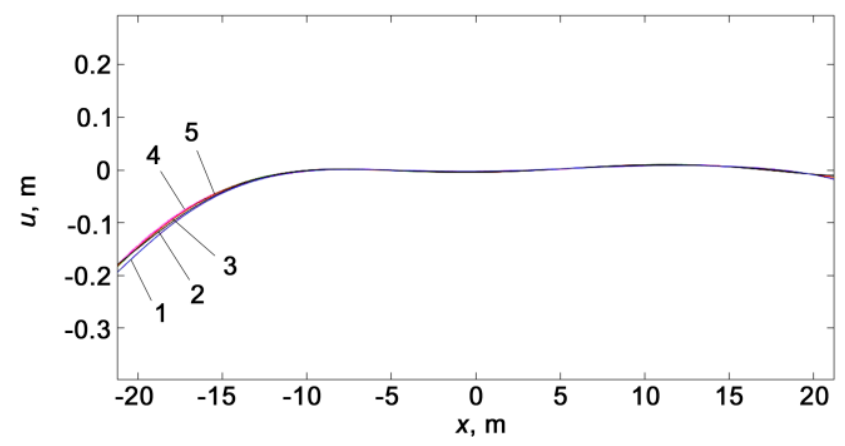

Fig. 5 Vertical displacements of the points along the rods length (general deformed view) at stiffness coefficients of the limiting supports: $1-c_{l}=c_{r}=12$ $\mathrm{MN} / \mathrm{m} ; 2-c_{l}=c_{r}=22 \mathrm{MN} / \mathrm{m} ; 3-c_{l}=c_{r}=32 \mathrm{MN} / \mathrm{m}$; $4-c_{l}=c_{r}=42 \mathrm{MN} / \mathrm{m} ; 5-c_{l}=c_{r}=120 \mathrm{MN} / \mathrm{m}$

\section{Conclusions}

There was developed an algorithm for solving differential equations with discontinuous boundary conditions for modeling structurally nonlinear vibrations of building structures. To improve the computational schemes for the practical problems implementation the Chebyshev's polynomials were used. There were avoided the computational difficulties and unacceptable discontinuities while simulating the oscillations of the simplified construction model of the floating bridge as a continuous system at the moment of the moving loading driving on and off the elastically supported system, and the smooth solutions were obtained.

It should be noted that to implement the general problem of modeling the floating bridge nonlinear vibrations at its continuous system with limiting supports at the ends under a moving loading, it is also necessary to use methods of modeling the vibrations of a moving car and single floating supports along with the developed algorithm.

The described technique can be easily used for modeling of structural-nonlinear vibrations of a wide range of building structures. This will allow to make an adequate prediction and evaluation of such structures behavior under a moving loading.

\section{References}

1. Telov, V. I.; Kartopoltsev, V. M. 1994. Road ferries and floating bridges. Tomsk: Tomsk University. 159 p. (in Russian).

2. Gridnev, S. Yu. 2008. Mathematic simulation of the behavior of beam systems under mobile load with regard to non-linearity, Privolzhsky Scientific Journal 2: 64-71 (in Russian).

3. Chen, X. J.; Lin, Z. M.; Wu, G. H.; Fu, S. X. 2006. Testing method and data analysis of moving-loadinduced dynamic displacements of floating bridges, Journal of Vibration, Measurement and Diagnosis 26(2): 97-101 (in Chinese).

4. Fu, S. X.; Cui, W. C.; Lin, Z. M.; Chen, X. J. 2004. Effect of gaps on static response of a belt floating bridge, Journal of Ship Mechanics 8(4): 86-94 (in Chinese).

5. Fu, S. X.; Cui, W. C.; Chen, X. J.; Wang, C. 2005. Hydroelastic analysis of a nonlinearly connected floating bridge subjected to moving loads, Marine Structures 18(1): 85-107.

http://dx.doi.org/10.1016/j.marstruc.2005.05.001.

6. Raftoyiannis, I. G.; Avraam, T. P.; Michaltsos, G. T. 2014. Analytical models of floating bridges under moving loads, Engineering Structures 68: 144-154. http://dx.doi.org/10.1016/j.engstruct.2014.03.002.

7. Zhang, J.; Miao, G. P., Liu, J. X., Sun, W. J. 2008. Analytical models of floating bridges subjected by moving loads for different water depths, Journal of Hydrodynamics, Ser. B. 20(5): 537-546. http://dx.doi.org/10.1016/S1001-6058(08)60092-X.

8. Wu, J. S.; Shih, P. Y. 1998. Moving-load-induced vibrations of a moored floating bridge, Computers \& Structures 66(4): 435-461. http://dx.doi.org/10.1016/S0045-7949(97)00072-2.

9. Kvåle, K. A.; Sigbjörnsson, R.; Øiseth, O. 2016. Modelling the stochastic dynamic behaviour of a pontoon bridge: A case study, Computers \& Structures 
165: 123-135.

http://dx.doi.org/10.1016/j.compstruc.2015.12.009.

10. Papaioannou, I.; Gao, R.; Rank, E.; Wang, C. M. 2013. Stochastic hydroelastic analysis of pontoon-type very large floating structures considering directional wave spectrum, Probabilistic Engineering Mechanics 33: $26-37$. http://dx.doi.org/10.1016/j.probengmech.2013.01.006.

11. Lee, J. H.; Seo, S. I.; Mun, H. S. 2016. Seismic behaviors of a floating submerged tunnel with a rectangular cross-section, Ocean Engineering 127: 32-47. http://dx.doi.org/10.1016/j.oceaneng.2016.09.033.

12. Wang, H. H.; Jin, X. L. 2016. Dynamic analysis of maritime gasbag-type floating bridge subjected to moving loads, International Journal of Naval Architecture and Ocean Engineering 8(2): 137-152. http://dx.doi.org/10.1016/j.ijnaoe.2015.11.002.

13. Albano, R.; Sole, A.; Mirauda, D.; Adamowski, J. 2016. Modelling large floating bodies in urban area flash-floods via a Smoothed Particle Hydrodynamics model, Journal of Hydrology 541A: 344-358. http://dx.doi.org/10.1016/j.jhydrol.2016.02.009.

14. Ufimtsev, E.; Voronina, M. 2016. Research of total mechanical energy of steel roof truss during structurally nonlinear oscillations, Procedia Engineering 150: 1891-1897. http://dx.doi.org/10.1016/j.proeng.2016.07.188.

15. Feygin, M. I. 1994. Forced oscillations of systems with discontinuous nonlinearities. Moscow: Nauka. 285 p. (in Russian).

16. Kargaudas, V.; Adamukaitis, N.; Žmuida, M. 2014. Natural oscillations of single span beam placed on cylindrical supports, Mechanika 20(3): 282-286. http://dx.doi.org/10.5755/j01.mech.20.3.6365.

17. Kirsanov, M. N. 2013. Static analysis of the cable system, Izvestiya MGTU "MAMI" t.1, 3(17): 89-93 (in Russian).

18. Gridnev, S. Yu.; Skalko, Yu. I.; Minaeva, N. V.; Yanaeva, V. V. 2019. Comparative analysis of models of limiting supports in the study of structurally nonlinear oscillations of elastically supported bar from mobile load, Journal of Physics: Conference Series 1203(1): 012030 .

http://dx.doi.org/10.1088/1742-6596/1203/1/012030.

19. Vladimirov, V. S. 1979. Generalized functions in mathematical physics. Moscow: Nauka, 379 p. (in Russian).

\section{S. Yu. Gridnev, Y. I. Skalko, A. O. Shimanovsky \\ MODELING OF CONSTRUCTIONS' \\ STRUCTURALLY NONLINEAR OSCILLATIONS USING CHEBYSHEV'S POLYNOMIALS}

S u m m a r y

A numerical algorithm for solving initialboundary value problems with nonlinear boundary conditions was developed and implemented. The algorithm is constructed with reference to modeling of oscillations of an elastically supported deformable rod with limit stops at the ends under the action of a moving variable force. Such a rod is the design scheme of a number of building structures, including the span structure of a floating bridge of continuous system with limiting rigid supports at the ends. Chebyshev's polynomials were used to improve the computational schemes for realizing the practical problems of modeling constructive-nonlinear oscillations of building structures. The solution does not lose stability for large values of the elasticity coefficients of elastic couplings. Using the developed approach, it is possible to perform virtual computing experiments to skip a variety of movable loads on the floating bridge to analyze its deformed state and to make well-grounded design decisions.

Keywords: floating bridge, constructive-nonlinear oscillations, Chebyshev's polynomials.

Received September 25, 2019 Accepted August 24, 2020 